\title{
Investigation of Protein Aggregation with a Bloch Surface Wave Sensor
}

\author{
Vincent Paeder*, Sara Santi, Lubos Hvozdara, Valeria Musi and Hans Peter Herzig \\ Optics and Photonics Technology Laboratory (OPT), Ecole Polytechnique Fédérale de Lausanne (EPFL), \\ Institute of Microengineering (IMT), Neuchâtel, Switzerland \\ vincent.paeder@epfl.ch
}

\begin{abstract}
We present a study of the dynamics of protein aggregation using a common path heterodyne Bloch surface wave label-free sensing scheme. We demonstrate the ability to detect, during thermal incubation, the first early dynamics events of protein aggregation and fibrillogenesis of amyloidbeta peptides, linked to Alzheimer's Disease. The calibration of the biosensor is made using Lysozyme protein, a well establish protein model system to examine structural and mechanistic principles. The sensor is able to detect the formation of small soluble aggregates at early stages of oligomerisation.
\end{abstract}

\section{INTRODUCTION}

A recent challenge for biophotonics techniques, already commonly used in sensing applications, is to develop and implement new diagnostic tools in different diseases and clinical settings. Here, we propose a new label-free optical platform based on Bloch Surface Waves [1] (BSW) sensitive to optical variations caused by conformational changes in proteins. In particular, this innovative and robust technology is applied to a topic that has become increasingly relevant in biomedical research: the early diagnosis of aberrant degenerative pathologies that are caused by the formation of amyloid fibrils (protein aggregates). An increasing number of pathologies have been associated with the formation of amyloid fibrils. Examples of such widespread pathologies, which have a large impact on human health since they are all currently incurable, are the Alzheimer and Parkinson diseases, Huntington's chorea, Creutzfeldt-Jacob disease, cystic fibrosis, Gaucher's disease, type II diabetes mellitus and the wide group of systemic amyloidosis [2].

It remains essential to develop new tools allowing early detection of the aggregates, since small oligomeric forms of aggregates are emerging as being the toxic species, suggesting their key role in disease pathogenesis [3]. It is imperative that new forms of diagnostics be developed to detect the disease prior to the onset of clinical symptoms. This will offer patients the prospect of preventive treatments that can block or significantly curb disease progression.

Here, we propose the use of an innovative optical method able to detect the early dynamic events of protein aggregation and fibrillogenesis of amyloid-beta peptides, linked to Alzheimer's Disease. The calibration of the biosensor is made using Hen Egg White Lysozyme (HEWL) protein, responsible for the hereditary non-neuropathic systemic amyloidosis. This protein has known structure and folding properties, it has been well characterized and shown to be useful as model system to examine structural and mechanistic principles [4]. Conclusions drawn on these molecules could therefore be confidently generalised to all amyloid fibrils (Fig. 1).

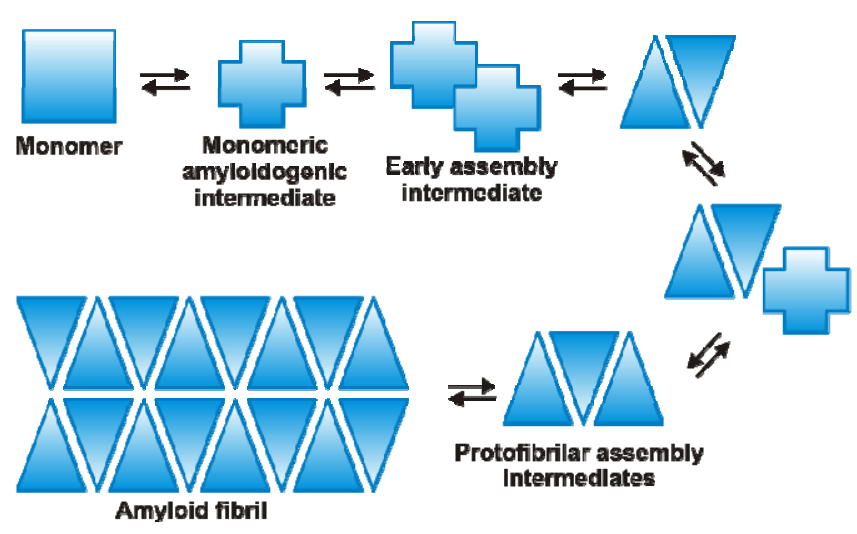

Figure 1. Schematic description of the process of amyloid formation (adapted from [5]) of a full length initially folded protein. The mechanism by which the different proteins gain a conformation, allowing monomers to bind to each other to form the regular amyloid fibril, has not yet been fully elucidated.

\section{EXPERIMENTAL METHOD}

The applied detection scheme is based on a common path heterodyne interferometer including a BSW sensor [6] (Fig. 2). TE and TM polarizations are separated after the sensor and used as reference and object channels, respectively. Such a configuration is allowed by the polarization-sensitive characteristics of the BSW, providing a high stability and a high sensitivity. The sensor is mounted on a rotating stage to track the resonance frequency of the BSW over large dynamic ranges.

BSW are surface waves supported at the surface of truncated periodic structures. A surface mode with a propagation constant below the light line in the external medium can be supported provided that a forbidden band exists in the underlying structure, which typically arises from periodicity. 


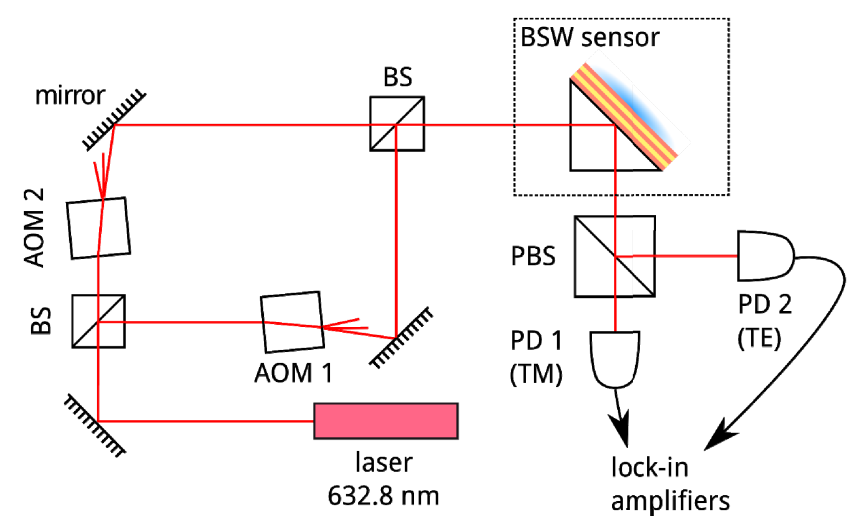

Figure 2. Principle of the common path heterodyne BSW sensing scheme

For the sake of convenience and simplicity, the structure used here is a $1 \mathrm{D}$ dielectric multilayer stack made of $\mathrm{SiO}_{2}$ (refractive index $\mathrm{n}_{\mathrm{L}}=1.46$ ) and $\mathrm{Si}_{3} \mathrm{~N}_{4}$ (refractive index $\mathrm{n}_{\mathrm{H}}=1.95$ ), and grown by PECVD (Oxford Plasmalab 80+). The designed stack supports a surface mode in TM polarization in an aqueous environment $(\mathrm{n}=1.333)$.

The BSW is excited in a Kretschmann configuration (Fig. 3). A temperature controlled microfluidic chamber is mounted atop the multilayer to incubate small amounts of solution. The evolution of the aggregation process is recorded over time during incubation.

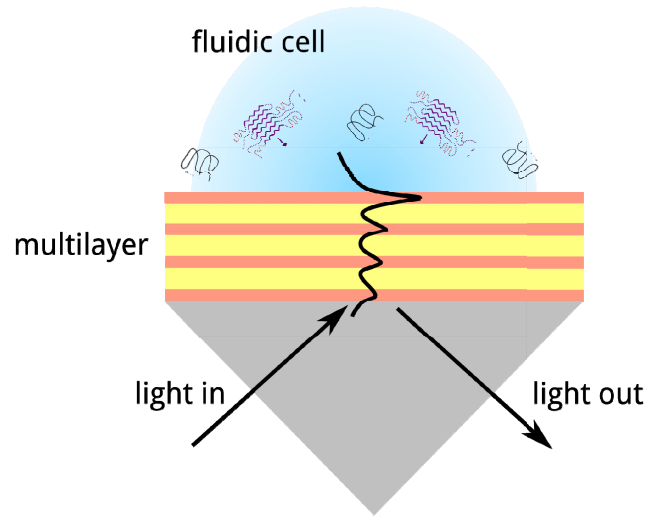

Figure 3. Excitation of a BSW in Kretschmann configuration

\section{RESULTS}

At first, the sensor has been characterized in static measurements (incubated outside the microfluidic cell) with HEWL in different concentrations (from $35 \mu \mathrm{M}$ to $14 \mathrm{mM}$ ). The sensor response relates the resonance angle with the concentration of remaining monomer. The proof of concept has been demonstrated and the results recently published [7].

The more recent results (manuscript in preparation) concern the kinetics studies of aggregation and conformational characterization of the Alzheimer's $A \beta 1-42$ peptides both by biochemical/biophysical classical methods and by using the BSW based sensor. The peptide aggregation during thermal incubation $\left(\mathrm{pH} 7,37^{\circ} \mathrm{C}\right)$ has been monitored using the classical techniques available in the laboratory (size exclusion chromatography, UV-visible, fluorescence and FTIR spectroscopies) (Fig. 4). The results are compared with the BSW based biosensor analysis of the samples incubated under the same conditions.
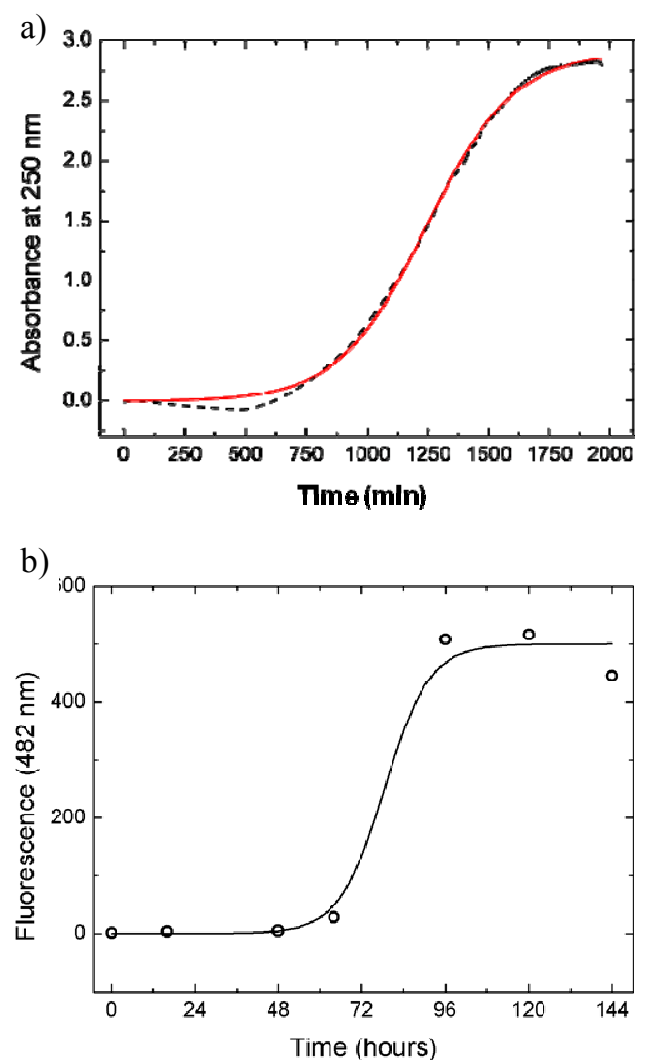

Figure 4. Biochemical and biophysical characterization of Abeta1-42 peptide during incubation at $37^{\circ} \mathrm{C}$ in $10 \mathrm{mM}$ Tris $\mathrm{HCl}$ buffer, $\mathrm{pH}$ 7.4. (a) UV-vis spectroscopy. Time-dependent absorbance of incubated A $\beta$ 1-42 sample monitored at $250 \mathrm{~nm}$ (black line). Red line: fitting curve obtained applying the nucleation-elongation kinetic model [8]. (b) Fluorescence. Time-dependent aggregation of $A \beta_{(1-42)}$ monitored by the fluorescence change of Thioflavin-T at $482 \mathrm{~nm}$. Open circles represent fluorescence data, the continuous black line is the curve obtained by fitting the experimental data with the equation describing the nucleationelongation kinetic model [8].

Both Hen Egg White Lysozyme (HEWL) and A $\mathrm{A1-42}$ molecules have been incubated for dynamics measurements into the microfluidic cell coupled to the BSW based biosensor using samples under the same conditions as for the classical detection. The time resolution measurements of 
during incubation of HEWL ( $\mathrm{pH} 2,65^{\circ} \mathrm{C}$ ) show that, in the current configuration (multilayer positioned vertically), aggregates tend to precipitate, causing a drop of the overall fluid density near the sensor surface. This is evidenced by a monotonous decrease of the resonance angle (Fig. 5, top).

On the other hand, incubation of amyloid- $\beta$ 1-42 $(\mathrm{pH} 7$, $37^{\circ} \mathrm{C}$ ), shows a different behaviour. Monomers and small aggregates are adsorbed at the surface and precipitate only after a certain aggregation threshold [9]. Evidence of this behaviour is shown in Figure 5 (bottom). A mathematical model for the sensor response has been derived to describe the interactions involving the formation of small soluble aggregates at the sensor surface (manuscript in preparation).
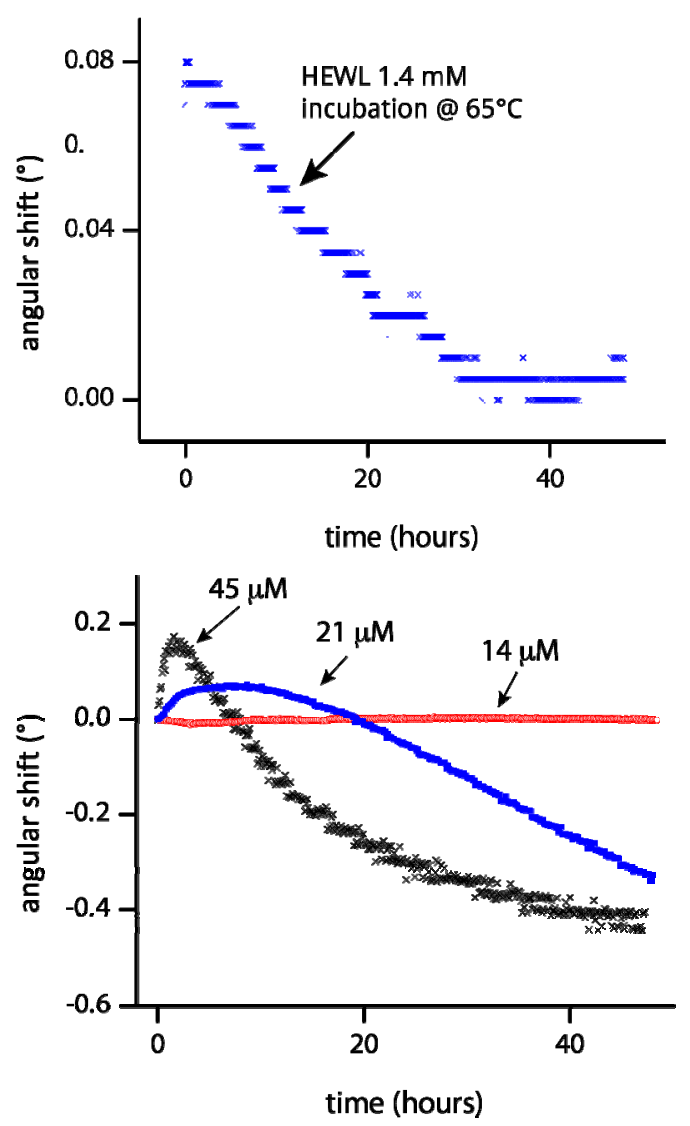

Figure 5. Time evolution of the BSW resonance angle during

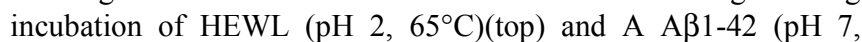
$37^{\circ} \mathrm{C}$ ) (bottom). Incubation conditions are detailed in Section 3.

\section{CONCLUSIONS}

We show in this study that a BSW-based heterodyne sensing scheme is suitable to investigate the dynamics of the aggregation processes of proteins. Time resolved measurements on Lysozyme and Alzheimer's beta amyloids show that the detection process relies on adsorption of monomers and small aggregates, as well as density depletion due to precipitation of large aggregates. A strong signal variation is observed in the early stage of incubation due to the interactions with the sensor surface during the aggregation process. Selective enhancement of the adsorption through surface functionalization is under active investigation. These results are of prime interest for the detection of conformational diseases at an early stage.

\section{REFERENCES}

[1] F. Giorgis, E. Descrovi, C. Summonte, L. Dominici, F. Michelotti, Experimental determination of the sensitivity of Bloch Surface Waves based sensors, Optics Express 18 (2010) 8087-8093.

[2] F. Chiti, C.M. Dobson, Protein misfolding, functional amyloid, and human disease, Annu Rev Biochem 75 (2006) 333-366.

[3] C.G. Glabe, Structural classification of toxic amyloid oligomers, J Biol Chem 283 (2008) 29639-29643.

[4] S.E. Hill, J. Robinson, G. Matthews, M. Muschol, Amyloid Protofibrils of Lysozyme Nucleate and Grow Via Oligomer Fusion, Biophys J 96 (2009) 3781-3790.

[5] J.W. Kelly, Towards an understanding of amyloidogenesis, Nat Struct Biol 9 (2002) 323-325.

[6] L.C. Su, R.C. Chen, Y.C. Li, Y.F. Chang, Y.J. Lee, C.C. Lee, C. Chou, Detection of prostate-specific antigen with a paired surface plasma wave biosensor, Analytical chemistry 82 (2010) 3714-3718.

[7] V. Paeder, V. Musi, L. Hvozdara, S. Herminjard, H.P. Herzig, Detection of protein aggregation with a Bloch surface wave based sensor, Sensors and Actuators B: Chemical 153 (2011).

[8] M.A. Watzky, A.M. Morris, E.D. Ross, R.G. Finke, Fitting yeast and mammalian prion aggregation kinetic data with the Finke-Watzky twostep model of nucleation and autocatalytic growth, Biochemistry 47 (2008) 10790-10800.

[9] S. Choi, Y. Yang, J. Chae, Surface plasmon resonance protein sensor using Vroman effect, Biosensors \& bioelectronics 24 (2008) 899-905. 